\title{
Erratum
}

\section{Synthesis of Advanced Intermediates of Lennoxamine Analogues}

Prajakta S. Sarang, Arun A. Yadav, Prashant S. Patil, Urlam Murali Krishna, Girish K. Trivedi,* Manikrao M. Salunkhe* Synthesis 2007, 1091.

In reference 8, the CCDC number for compound 1c was incorrectly cited. The correct number is CCDC 633471. 